\title{
Orbital elements of 9 visual double stars ${ }^{\star}$
}

\author{
A. Alzner \\ Zeckerner Hauptstr. 3, D-91334 Hemhofen, Germany
}

Received April 22; accepted April 24, 1998

\begin{abstract}
Orbital elements and ephemerides for 9 visual double stars are given.
\end{abstract}

Key words: binaries: visual — astrometry

\section{Orbital elements, dynamical parallaxes and masses} (Table 1)

\section{Ephemerides (Table 2)}

\section{Residuals (Table 3-available electronically)}

\section{Notes}

The Thiele - van den Bos method was used for the preliminary orbits of ADS7929 and ADS15215. For the calculation of the other stars least square fits were performed using differential corrections in polar coordinates (Heintz 1967).

1. ADS2446 = STT53. The best of the older orbits is that by Rabe (1948), but the motion is now ahead of his prediction. The orbit by Scardia (Circular 82) has a too short period and leaves a large residual to van Biesbroeck's observation 1924.76. The orbit by Zulevic (Circular 93) gives large residuals for all observations from 1923.78 to 1933.16. The dynamical parallax 0.017 is somewhat larger than the parallax measured by Hipparcos (13.94 mas).

2. STT89, not in the ADS-catalogue. Recalculated because Baize'orbit 1981 gives distances of 0.29 to 0 ". 30 in the years 1896 to 1900 when the star was found single by Aitken and Hussey with the $36^{\prime \prime}$-Lick-refractor. These negative results and recent distance measurements give a higher excentricity.

3. ADS4373 = HU39. First orbit for this somewhat puzzling pair which has finished almost two revolutions

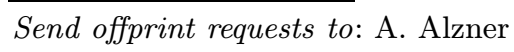

* Table 3 is available in electronic form only, via the CDS at 130.79.128.5 or via http://cdsweb.u-strasbg.fr/Abstract.html since discovery. Bryants measurement 1906 is given here but was not used for the calculations because it does not fit to any variant. The high excentricity variant leaves an unusually large residual to Aitkens measurements 1922. Putting the 1920s observations and Worleys measurement 1975 into the 3rd quadrant a better fit with a low excentricity orbit results with two main sequence stars.

4. ADS6291 = STF1130. This binary was recalculated, because Baize's orbit from 1984 now shows large negative residuals in the position angles. For the differential correction many iterations were necessary, as the new period is much longer and only 3 or 4 elements could be corrected simultaneously during one iteration. Periastron is passed, but the elements are still weakly determined.

5. ADS7929 = STT229. First calculation after van Biesbroecks provisional circular orbit. Consistent measures, but the elements are still preliminary. As ADS7929 is a subgiant pair, no dynamical parallax was calculated.

6. ADS11584 = STT363. The CHARA Speckle observations near periastron have allowed a recalculation after Zulevic's two preliminary orbits.

7. ADS11971 = STF2434BC. No speckle and no Hipparcos measurements. Calculated without knowing the orbit of Popovic \& Catovic (1994) which does not seem to fit the observations well. My elements are, however, preliminary as well. Better fits to the angles leave larger residuals to the distances and vice verse. I have estimated a brightness difference of 1.0 mags during the measurements with a $360 \mathrm{~mm}$ Newtonian (1995).

8. $\operatorname{ADS} 14073=$ BU151. The rapid binary $\beta$ Del was recalculated, because recent Speckle measurements show positive residuals in the angles to the orbit by Hartkopf et al. (1989). On the other hand their orbit gives systematic positive angle residuals to many of the early visual measurements. When trying to do differential corrections in all seven Campbell elements, no satisfactory orbit for both, the Speckle and the visual measurements could be obtained, as the visual observations have rendered the semi major axis too large. In a first step, all Speckle measurements plus H.M. Jeffers interferometric 
Table 1.

\begin{tabular}{llllllllllll}
\hline $\begin{array}{l}\text { ADS or } \\
\text { Name }\end{array}$ & $m_{A}, m_{B}$ & Sp. & $P$ & $T$ & $a$ & $e$ & $i$ & $\omega$ & $\Omega_{2000}$ & $p_{\text {dyn }}$ & mass \\
\hline 2446 & $7.9,8.5$ & G0 & 113.5 & 1928.31 & 0.53 & 0.76 & 137.2 & 46.4 & 118.4 & 0.017 & 2.2 \\
STT89 & $6.4,7.4$ & A1 & 139 & 1918 & 0.39 & 0.71 & 83.7 & 48.1 & 113.5 & 0.009 & 4.6 \\
4373 & $8.4,8.5$ & G0 & 47.5 & 1978.6 & 0.29 & 0.28 & 95.8 & 215.9 & 43.3 & 0.017 & 2.1 \\
& & & 50.0 & 1981.4 & 0.19 & 0.95 & 106.0 & 187.6 & 44.1 & 0.010 & 2.8 \\
6291 & $8.9,9.4$ & G0 & 1000 & 1981.4 & 1.88 & 0.81 & 53.0 & 168.5 & 147.1 & 0.016 & 1.6 \\
7929 & $7.4,7.8$ & A3IV & 320 & 2072.4 & 0.626 & 0.485 & 159.0 & 286.2 & 63.3 & - & - \\
11584 & $7.6,7.8$ & F0 & 335 & 1986.35 & 0.73 & 0.90 & 78.6 & 68.4 & 161.0 & 0.010 & 3.3 \\
11971 & $8.7,10.6$ & G5 & 402.7 & 2007.6 & 1.195 & 0.562 & 145.7 & 200.3 & 102.6 & 0.019 & 1.6 \\
14073 & $4.1,5.0$ & F5IV & 26.65 & 1962.87 & 0.443 & 0.355 & 62.1 & 348.8 & 177.9 & - & 3.26 \\
15215 & $8.4,9.4$ & G0 & 264 & 2029 & 0.52 & 0.555 & 122.1 & 350.2 & 46.8 & 0.009 & 2.6 \\
\hline
\end{tabular}

Table 2.

\begin{tabular}{|c|c|c|c|c|c|c|}
\hline $\begin{array}{l}\text { ADS or } \\
\text { Name }\end{array}$ & & 1998.0 & 2000.0 & 2002.0 & 2004.0 & 2006.0 \\
\hline 2446 & & $251.8,0.746$ & $250.3,0.731$ & $248.6,0.714$ & $246.9,0.696$ & $245.1,0.677$ \\
\hline STT89 & & $302.3,0.384$ & $302.7,0.369$ & $303.2,0.354$ & $303.7,0.338$ & $304.3,0.322$ \\
\hline 4373 & $e=0.28$ & $41.6,0.349$ & $40.6,0.333$ & $39.3,0.307$ & $37.9,0.271$ & $35.9,0.226$ \\
\hline & $e=0.95$ & $43.4,0.343$ & $43.1,0.354$ & $42.7,0.362$ & $42.4,0.366$ & $42.1,0.368$ \\
\hline 6291 & & $15.5,0.373$ & $21.9,0.377$ & $28.1,0.384$ & $34.1,0.394$ & $39.7,0.405$ \\
\hline 7929 & & $270.5,0.718$ & $269.1,0.709$ & $267.7,0.701$ & $266.2,0.691$ & $264.7,0.682$ \\
\hline 11584 & & $345.2,0.328$ & $346.0,0.357$ & $346.8,0.383$ & $347.4,0.406$ & $348.0,0.427$ \\
\hline 11971 & & $295.1,0.555$ & $289.3,0.545$ & $283.4,0.536$ & $277.2,0.528$ & $270.8,0.519$ \\
\hline 14073 & & $331.4,0.416$ & $342.1,0.516$ & $349.7,0.578$ & $356.4,0.596$ & $3.1,0.568$ \\
\hline 15215 & & $181.4,0.287$ & $177.2,0.266$ & $172.2,0.245$ & $166.2,0.225$ & $159.3,0.207$ \\
\hline
\end{tabular}

measurements were used to calculate a preliminary orbit. Couteau's orbit from 1962 served as a starting point. Several more iterations now using all measurements corrected six elements, the semi major axis a being fixed. The period of the new orbit is exactly the same as calculated by Couteau, the total mass is $3 \%$ smaller than that calculated from Hartkopf et al.'s elements using the Hipparcos parallax. As ADS14073 is a subgiant pair, the formula for the dynamical parallax is not applicable.

9. ADS15215 = STT448. First, preliminary orbit. The arc of the companion has covered only 60 degrees in the 150 years since the discovery but the curvature gives already an idea of the ellipse. A complete revolution will probably be finished in less than the following 150 years. Having been closed in since more than 90 years this pair has become a difficult object for my $360 \mathrm{~mm}$ Newtonian. Following a proposal by W.D. Heintz half of the correction of the angles has been given to the angles of the O. Struve measures. The result leaves the question open whether these are two main sequence stars or possibly subgiants.
Acknowledgements. The author wishes to thank Prof. W.D. Heintz from the Swarthmore College for his help and many advices especially concerning orbit calculations using the techniques of differential corrections.

\section{References}

Heintz W.D., 1967, Acta Astron. 17, 311

Rabe W., 1948, Astron. Nachr. 276, 265

Baize P., 1981, A\&AS 44, 199

Baize P., 1984, A\&AS 56, 103

Van Biesbroeck G., 1904, Ann. Obs. R. de Belg. 9, 107

Abt H.A., 1981, ApJS 45, 437

Zulevic D.J., 1975, Circ. Inf. No. 66

Popovic G.M., Catovic Z., 1994, Bull. Astron. Belgrade 149, 95

Couteau P., 1962, J. Obs. 45, 39

Hartkopf W.I., McAlister H.A., Franz O.G., 1989, AJ 98, 1014

Heintz W.D., 1978, Double Stars. D. Reidel Publishing Comp. p. 24 